\title{
Strain-Directed Layer-By-Layer Epitaxy Toward van der Waals Homo- and Heterostructures
}

Yi Wan, Jing-Kai Huang, Chih-Piao Chuu, Wei-Ting Hsu, Chien-Ju Lee, Areej Aljarb, Chun-Wei Huang, Ming-Hui Chiu, Hao-Ling Tang, Ci Lin, Xuechun Zhang, Ching-Ming Wei, Sean Li, Wen-Hao Chang, Lain-Jong Li,* and Vincent Tung*

Cite This: ACS Materials Lett. 2021, 3, 442-453

Read Online

\section{ACCESS | Lل|ll Metrics \& More | 回 Article Recommendations | sl Supporting Information}

ABSTRACT: Transition-metal dichalcogenide (TMDC) homoand heterostacks hold tantalizing prospects for being integrated as active components in future van der Waals $(\mathrm{vdW})$ electronics and optoelectronics. However, most TMDC homo- and heterostacks are created by onerous mechanical exfoliation, followed by a mixing-and-matching process. While versatile enough for pilot demonstrations, these strategies are clearly not scalable for practical technologies and widespread implementations. Here, we report a two-step epitaxy strategy that promotes the growth of second-layer TMDCs on the basal plane of the first TMDCs epilayer. The first-layer TMDCs are grown on substrates where the tensile strength can be tuned by the
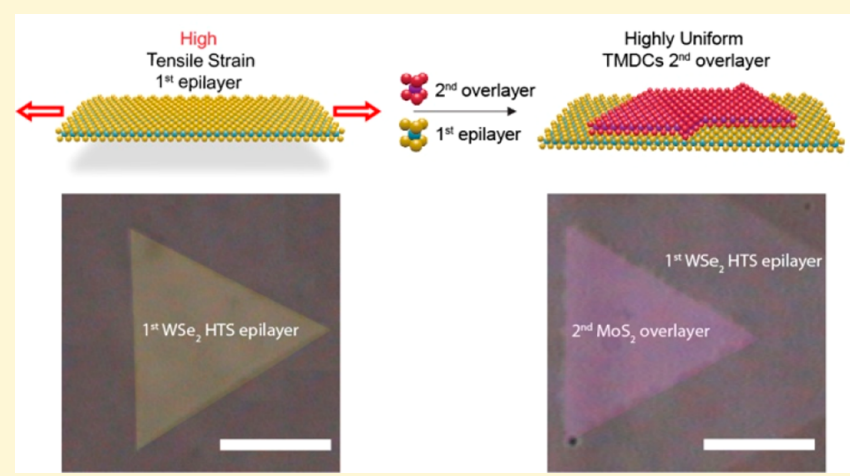
control of chemical environments. The succeeding epilayers then prefer to grow layer-by-layer on the highly tensile-strained first layers. The result is the growth of high-density TMDC homo $\left(\mathrm{WSe}_{2}\right)$ bilayers and hetero $\left(\mathrm{WSe}_{2}-\mathrm{MoS}_{2}\right)$ bilayers with an exceedingly high yield ( $>99 \%$ bilayers) and uniformity. A density functional theory simulation further sheds light on how strain engineering shifts the subsequent layer growth preference. Second-harmonic generation and high-angle annular dark-field scanning transmission electron microscopy collectively attest to the $\mathrm{AB}$ and $\mathrm{AA}^{\prime}$ stacking between the TMDC epi- and overlayers. The proposed strategy could be a versatile platform for synthesizing diverse arrays of vdW homo- and heterostacks, thus providing prospects for realizing largescale and layer-controllable two-dimensional electronics.

Two-dimensional (2D) van der Waals (vdW) structures embody a unique class of acritical lattices in which material properties can be manipulated via varying the composition, stacking orders, and relative orientation across the atomically thin junctions. Building such artificial vdW junctions is made possible by disassembling bulk crystals into $2 \mathrm{D}$ monolayers and reassembling them into bilayer artificial lattices, yielding access to a plethora of exciting physics. ${ }^{1,2}$ The variety of artificial homo- and heterostructures can be further extended when combined with the recent rediscoveries of transitionmetal dichalcogenides (TMDCs), therefore providing a route to previously unachievable semiconductor junctions, superlattices, and light-matter interactions. ${ }^{3}$ Notable discoveries include but are not limited to the advent of atomically thin $\mathrm{p}-$ $\mathrm{n}$ junctions for the ultimate functional unit for nanoscale electronic and optoelectronic devices, ${ }^{4-6}$ exceedingly high density of states that induce exceptionally high drive currents in the ballistic limit, ${ }^{7,8}$ enhanced light absorption with a wider spectral response in UV and near-infrared (NIR) regions, ${ }^{9,10}$ and prominent mechanical durability implemented in flexible devices. $^{11}$ Despite the immense interest and continuing experimental success by directly mixing and matching monolayer flakes of different materials, a widespread implementation of vdW homo- and heterostacks has yet to occur. This is primarily due to the difficulty of identifying a general, scalable, and reliable epitaxy mechanism that drives the direct growth of vdW homo- and heterostacks with control

Received: November 30, 2020

Accepted: February 22, 2021 
(a)

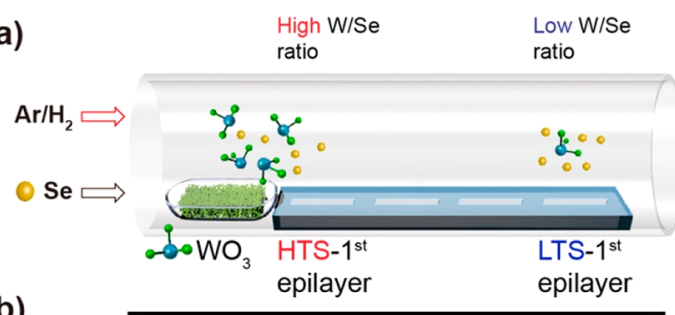

(b)
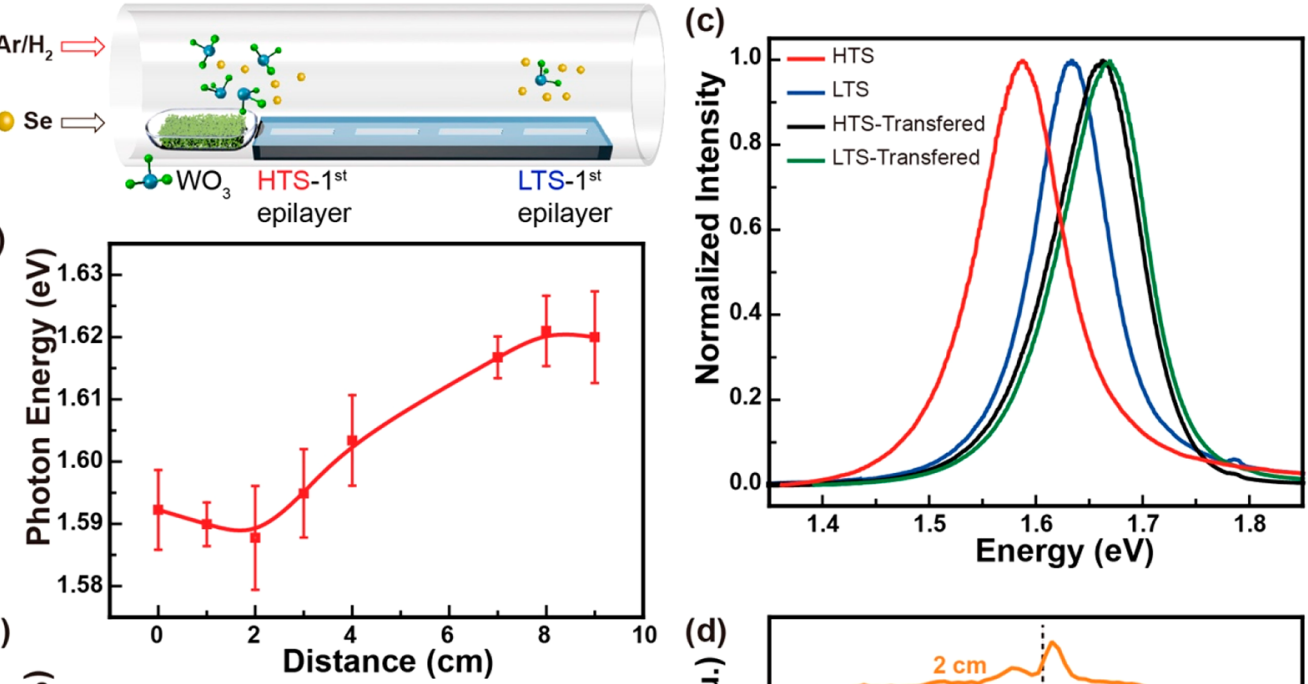

(e)
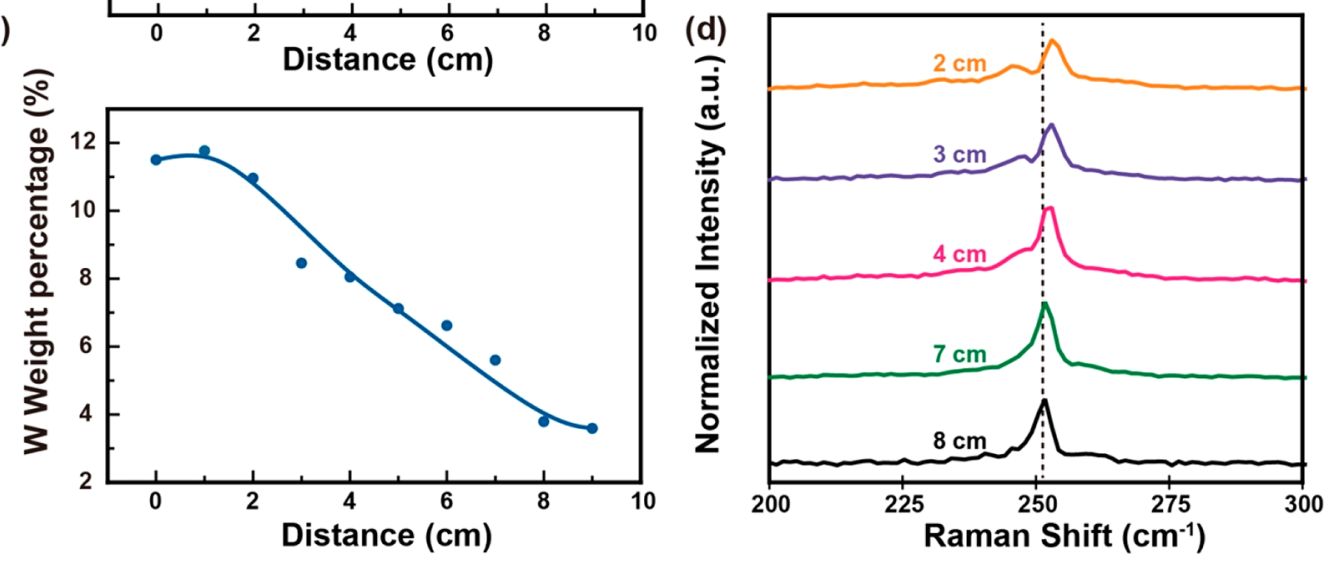

Figure 1. Strain engineering of the first WSe $e_{2}$ epilayers. (a) Schematic illustration shows a representative CVD epitaxy growth of the first $W_{2} e_{2}$ epilayers. (b) Photon energy distribution of the first WSe $e_{2}$ epilayers collected at different distances away from the $\mathrm{WO}_{3}$ source. Error bars are included to provide a standard deviation of photon energy. Data were collected from over 50 samples. (c) PL spectra of HTS and LTS first WSe $e_{2}$ epilayers grown on sapphire substrates before and after transferring at room temperature. (d) Raman spectra of the as-grown first $\mathrm{WSe}_{2}$ epilayers collected at different distances from away the $\mathrm{WO}_{3}$ source. (e) The concentration distribution of $\mathrm{W}$ (blue) as a function of different distances away from the $\mathrm{WO}_{3}$ source. The weight percentage of $\mathrm{W}$ with respect to detected elements ( $\mathrm{Al}, \mathrm{O}$, and $\left.\mathrm{W}\right)$ based on EDX data.

over bilayer coverage and content in tandem with the preservation of crystal qualities.

The general epitaxy growth in chemical vapor deposition (CVD) usually involves the vaporization of precursors, nucleation of TMDC seeds, and the stitching of individual seeding flakes. In this light, the vertical growth of homo- and heterostructures shall, in principle, be accomplished through the successive growth of a second material on the basal plane of a first layer. Currently, significant efforts have been reported to grow few-layered TMDCs in an epitaxial fashion. ${ }^{12-17}$ Ye et al. ${ }^{12}$ systematically examined the thermodynamic and kinetic controls of the vertically stacked $2 \mathrm{D}$ materials, where the growing conditions, such as annealing temperatures and adatom flux, play crucial roles governing the final structure. Similarly, Li et al. ${ }^{13}$ demonstrated that the active clusters with a high diffusion barrier, for example, the ratio between metal oxides and chalcogenides, would modulate the heteroepitaxy growth direction. Besides, a wide variety of growth promoters, including aromatic derivatives and alkali metal halides, has been implemented to regulate the direction of the growth front resulting from the enhanced diffusivity of transition-metal oxide precursors. In a nutshell, this research directed at understanding the mechanisms that underly the epitaxial growth of vdW homo- and heterostructures predominately hinge on two aspects. One is the thermodynamic control of nucleation on the first TMDC epilayer, and the other is the kinetic modulation of the growth front of the second TMDC overlayer. $^{13-15}$ However, most discussion is focused on the way to overcome the exceedingly high activation energy on the basal plane of the first epilayer; little work expounds on what properties of the first epilayer will result in a different transition in the growth mode of succeeding TMDC layers.

Here we report the modulation of growth modes of succeeding TMDC layers through the creation of strain fields of the first epilayer during the epitaxy growth. The strain level of the first epilayer was engineered by the dissimilar diffusivity of metal oxide and chalcogen precursors. Comprehensive density functional theory (DFT) calculations indicate that built-in strain fields overshadow the activation energy that predominately dictates the successive growth mode of the epilayer. Atomically resolved images show that the second overlayer grown on top of the strained first epilayer has the propensity to follow both $\mathrm{AA}^{\prime}(2 \mathrm{H})$ and $\mathrm{AB}(3 \mathrm{R})$ stacking. Since the strain on TMDCs can be built on substrates in the CVD growth and is not limited to the current material combinations, the finding here represents a very visible nexus 
between epitaxial growth and advanced nanoengineering that has the potential to open a new opportunity in the scalable production of TMDC homo- and heterostacks.

Results and Discussion

Strain on single-layer $\mathrm{WSe}_{2}$. The process for activating the basal plane as the growth front begins with the epitaxial growth of $\mathrm{WSe}_{2}$ monolayers by CVD that features the dual-heating zones $^{18}$ as schematically illustrated in Figure 1a. Here, the $\mathrm{WO}_{3}$ source was located at the center of heating zone 2 , and sapphire substrates were placed adjacent to the $\mathrm{WO}_{3}$ boat. The temperature of heating zone 2 was fixed at $900{ }^{\circ} \mathrm{C}$. The annealing temperature of heating zone 1 , where Se powders are located, varied from 220 to $300{ }^{\circ} \mathrm{C}$. We found that this Se annealing temperature is strongly associated with the shift in photoluminescence (PL) of the as-grown $\mathrm{WSe}_{2}$ monolayer specimens that is characteristic of the structural strain (Figure S1). ${ }^{19-22}$ When the Se annealing temperature was set at 220 ${ }^{\circ} \mathrm{C}$, the as-grown $\mathrm{WSe}_{2}$ monolayer shows an average $\mathrm{PL}$ emission of $\sim 1.56 \mathrm{eV}$ along the whole deposition distance. Conversely, the PL energy shifts to $1.63 \mathrm{eV}$ when the Se annealing temperature is set at $300{ }^{\circ} \mathrm{C}$. To this end, we set the annealing temperature of the first hot zone at a medium temperature of $270^{\circ} \mathrm{C}$, which in turn enables us to explore the origin of built-in strain fields. PL spectra were taken from $\mathrm{WSe}_{2}$ specimens epitaxially grown on sapphire substrates located between the upstream and downstream regions as shown in Figure $1 \mathrm{~b}$. The distance between each sapphire substrate is evenly separated by $1 \mathrm{~cm}$ to ensure the comparison of PL spectra and the associated built-in strain fields on a fair footing. To better understand the correlative strain effect, we selected two typical samples at the opposite ends of depositing position profiles as shown in Figure 1c. Relative to the prototypical emission of $\sim 1.66 \mathrm{eV}$ of an unstrained monolayer $\mathrm{WSe}_{2}$ reported in the literature, ${ }^{20} \mathrm{PL}$ spectra of $\mathrm{WSe}_{2}$ collected near the beginning of the diffusion pathway marked as $0 \mathrm{~cm}$ displayed a substantial red shift of $74 \mathrm{meV}$, which can be translated into a tensile strain of $\sim 1.2 \%$, designated as hightensile-strained (HTS) $\mathrm{WSe}_{2} \cdot{ }^{23,24}$ Meanwhile, only a minor red shift of $27 \mathrm{meV}$ was observed from $\mathrm{WSe}_{2}$ specimens grown near the end of the diffusion pathway, where Se vapors become predominant. The minor shift accounts for a relatively low tensile strain of $\sim 0.43 \%$, namely, low-tensile-strained (LTS) $\mathrm{WSe}_{2} \cdot{ }^{23,24}$ Intriguingly, it is found that the tensile strains in both samples relaxed after the wet transfer to freshly cleaned sapphires and their emission peaks revert back to $\sim 1.66 \mathrm{eV}$. The tensile strain is likely built upon the cooling after growth, and it is proposed that the different strains might be related to the thermal coefficient difference between the sapphire substrate and the TMDCs grown with various conditions, which will be further discussed in the following context.

It is known that defects, trions, and band-to-band emissions all exhibit characteristic emission evolutions when excited at low temperatures. ${ }^{25-27}$ Thus, to rule out the defect- and doping-induced shift in PL emission, we performed lowtemperature PL measurements as shown in Figure S2. As suggested in the Gaussian fitted spectra, we did not observe a distinctive PL emission difference that stemmed from defect and doping contributions of both HTS and LTS $\mathrm{WSe}_{2}$ samples. These results further attest to the fact that the peak position and intensity alteration are more likely caused by a strain-induced band variation. (See the detailed discussion in the Supporting Information, Note S1.) Furthermore, on the basis of temperature-dependent photoluminescence measure- ments (Figures S3 and S4), we noted that the PL energies of both samples all displayed a large redshift with increasing temperature, as a result of the increased electron-phonon interactions and the varied bonding lengths. The HTS sample can be considered that the $\mathrm{WSe}_{2}$-sapphire exhibits a stronger interaction, while the low-tensile-strained sample LTS exhibits a much weaker (van der Waals-like) interaction. The mismatch in the thermal expansion coefficient between the first epilayer $\left(\mathrm{WSe}_{2},(1.1-1.4) \times 10^{-5} / \mathrm{K}\right)$ and underlying substrate (sapphire, $\left.(5-8.3) \times 10^{-6} / \mathrm{K}\right)$ has profound impacts in the HTS-WSe ${ }_{2}$ when the temperature of the PL measurement was decreased from 300 to $4 \mathrm{~K} .{ }^{28}$ The result is that the much wider PL energy difference between the HTS and LTS samples increased from $40 \mathrm{meV}(300 \mathrm{~K})$ to $\sim 60 \mathrm{meV}(4 \mathrm{~K})$. To this end, we employed a modified Varshni relationship to quantitatively investigate the temperature dependence on the bandgap of strained $\mathrm{WSe}_{2}{ }^{29,30}$

$$
E_{\mathrm{g}}(\mathrm{T})=E_{\mathrm{g}}(0)-S\langle\hbar \omega\rangle\left[\operatorname{coth}\left(\frac{\langle\hbar \omega\rangle}{2 k_{\mathrm{B}} T}\right)-1\right]
$$

where $E_{\mathrm{g}}(0)$ is the bandgap energy at absolute zero temperature, $S$ is a dimensionless constant that represents the strength of the exciton-phonon coupling, $\langle\hbar \omega\rangle$ describes the average phonon energy involved in electron-phonon interactions, $\hbar$ is Planck's constant, and $k_{\mathrm{B}}$ is the Bolzmann constant. For HTS-WSe $\mathrm{H}_{2}$, the fitting parameter was extracted with $E_{\mathrm{g}}(0) \approx 1.67 \mathrm{eV}, S \approx 2.07$, and $\langle\hbar \omega\rangle \approx 11.71 \mathrm{meV}$. A similar fitting to LTS-WSe $_{2}$ yields $E_{\mathrm{g}}(0) \approx 1.73 \mathrm{eV}, S \approx 2.66$, and $\langle\hbar \omega\rangle \approx 18.80 \mathrm{meV}$. By comparing these parameters, the HTS sample was found to exhibit a lower bandgap value and weaker exciton-phonon coupling, agreeing well with the previously reported results where monolayer $\mathrm{WSe}_{2}$ is subjected to a tensile strain. ${ }^{31}$ Meanwhile, Raman spectroscopy was used to study the spatial distribution of the built-in strain of the asgrown $\mathrm{WSe}_{2}$. Figure $1 \mathrm{~d}$ demonstrates that the $\mathrm{E}_{2 \mathrm{~g}}$ peak of $\mathrm{WSe}_{2}$ splits into two peaks and shifts to opposite directions when the growth of $\mathrm{WSe}_{2}$ happens near the upstream. This is characteristic of the emergence of a relatively higher tensile strain, ${ }^{21}$ which is also typically seen in CVD-grown single crystal TMDCs. ${ }^{32,33}$ Meanwhile, when the growth of $\mathrm{WSe}_{2}$ takes place at the downstream region, the splitting of the doublet peak gradually narrows and eventually merges at $\sim 250$ $\mathrm{cm}^{-1}$, indicative of a nearly strain-free state. ${ }^{21}$ These results collectively attest to the fact that the peak position and intensity alteration are more likely caused by a strain-induced band variation during the epitaxy.

As mentioned in the previous section, the root cause responsible for the strain variation is the combined results of subtle differences in growth environments. On the one hand, it is known that the diffusion of metal (oxide) vapors in conjunction with a significant vapor concentration drop always gives rise to a nonuniform growth distribution when the distance between metal oxide sources and designated substrates increases. ${ }^{34}$ On the other hand, Se exhibits a low melting point $\left(220^{\circ} \mathrm{C}\right)$ and thus can diffuse throughout the whole furnace tube when the furnace temperature reaches 900 ${ }^{\circ} \mathrm{C}$ at 10 Torr. ${ }^{34} \mathrm{We}$ performed the evaporation of $\mathrm{WO}_{3}$ powders in a standard growth condition except for the absence of Se (see Figure S5 for SEM images). An energy-dispersive Xray analysis (EDX) is used to examine the relative $\mathrm{W}$ weight percentage of the deposited film along the path of diffusion. Figure 1e clearly shows that the concentration profile of $\mathrm{W}$ 


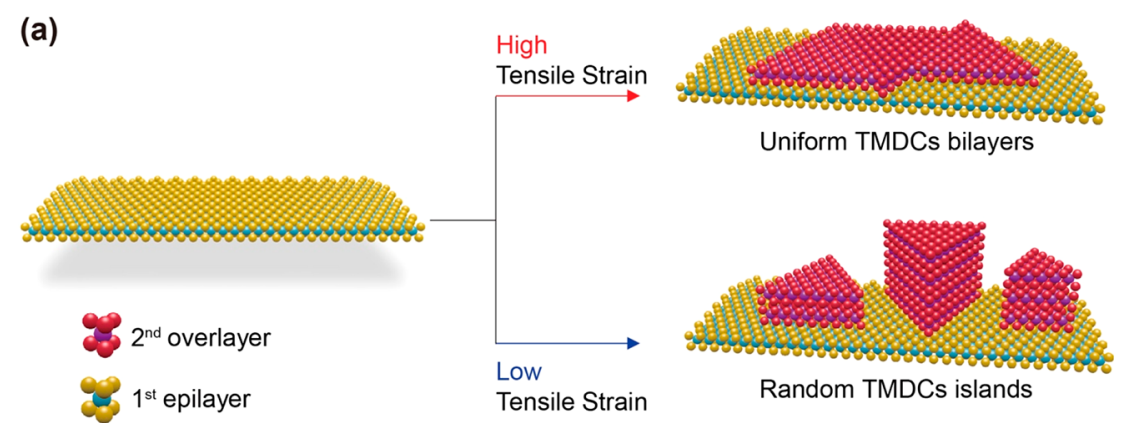

(b)

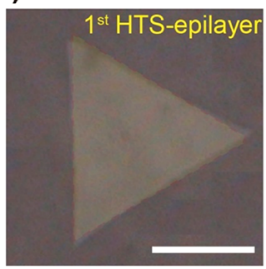

(e)

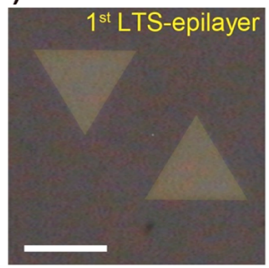

(c)

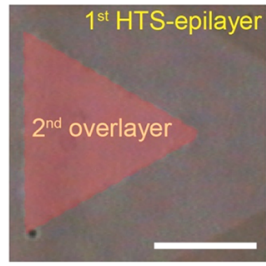

(f)

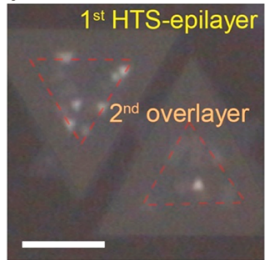

(d)

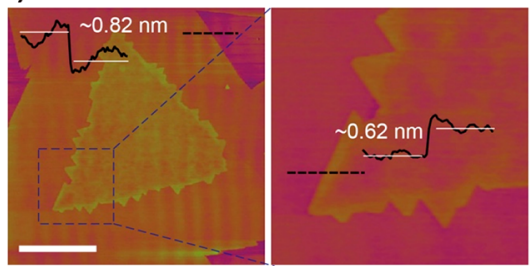

(g)

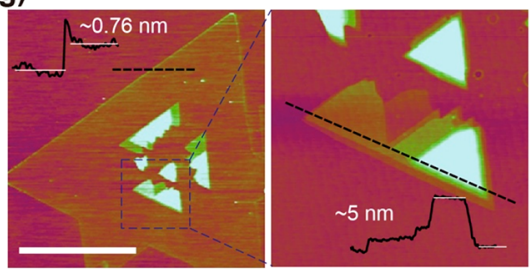

Figure 2. Growth-mode control by tensile strain. (a) Schematic representation features the growth of homogeneous second WSe $e_{2}$ overlayers on the first HTS-WSe $e_{2}$ epilayer in a layer-by-layer fashion. Meanwhile, island growth of multilayered clusters was found on the basal plane of the LTS-WSe $e_{2}$. (b) OM image of the as-grown $(0 \mathrm{~cm}) \mathrm{HTS}-W \mathrm{We}_{2}$ monolayer. (c) OM showing the formation of WSe $\mathrm{e}_{2}$ homobilayer indicated by the red, dashed triangle. (d) Corresponding AFM images of $\mathrm{WSe}_{2}$ homobilayers as well as a close-up view of the blue-marked area. (inset) The height profile along the black dotted line. (e) OM image of the as-grown $(9 \mathrm{~cm}) \mathrm{LTS}$-WSe $\mathrm{e}_{2}$ monolayer. (f) $\mathrm{OM}_{\text {of }} \mathrm{WSe} \mathrm{e}_{2}$ islands formed during the second growth. $(\mathrm{g})$ Corresponding AFM images reveal the multilayered islands scattered on the basal plane of the first LTS-WSe ${ }_{2}$ epilayers. Scale bars: $5 \mu \mathrm{m}$.

distributes in a declining fashion, suggesting that the $\mathrm{WO}_{3} / \mathrm{Se}$ vapor ratio scales disproportionally with the increasing distance between growth location and $\mathrm{WO}_{3}$ source. This concentration profile shows a similar trend compared to the energy distribution shown in Figure 1b. In the meantime, we also performed the first $\mathrm{WSe}_{2}$ epilayer growth on top of $\mathrm{SiO}_{2} / \mathrm{Si}$ substrates as described in Figure S6 and Supporting Information, Note S4. On the basis of the experimental observations and spectroscopic characterizations, we arrived at the conclusion that the $\mathrm{WO}_{3} / \mathrm{Se}$ vapor concentration profile enables the modulation of the strain levels in the monolayer $\mathrm{WSe}_{2}$. Under the Se-deficient atmosphere (growth takes place near the $\mathrm{WO}_{3}$ boat or at low Se heating temperature), the reduced $W$ atoms may not be fully selenized and then completely converted into $\mathrm{WSe}_{2}$. Hence, fractions of $\mathrm{W}$ atoms in the $\mathrm{WSe}_{2}$ flake are chemically tethered with the oxygenterminated sapphire surfaces at the reaction temperature. Next, the chemically pinned $\mathrm{WSe}_{2}$ tends to develop the high built-in strain by virtue of the mismatch of the thermal expansion coefficient between that of $\mathrm{WSe}_{2}$ and sapphire when cooling down from a high annealing temperature in the growth process. ${ }^{28}$ At the same time, the Se-rich environment efficiently transforms $\mathrm{WO}_{3}$ precursors to $\mathrm{WSe}_{2}$, where the unsaturated $\mathrm{W}-\mathrm{O}$ bond formation enables $\mathrm{WSe}_{2}$ monolayers to find an energetically favorable configuration and thus leads to a lowtensile-strained monolayer. We note that the deposition temperature near the upstream location is determined to be
$925{ }^{\circ} \mathrm{C}$, and it gradually decreases to $892{ }^{\circ} \mathrm{C}$ when the depositing position moves to the downstream site. (Table S1). As discussed in Supporting Information, Note S3, the temperature difference measured at 0 and $9 \mathrm{~cm}$ would be a nuance $(\sim 0.03 \%)$ and less likely to contribute to the built-in strain on the first $\mathrm{WSe}_{2}$ epilayer.

Strain-induced epitaxy of $\mathrm{WSe}_{2}$ homobilayers. To elucidate how a tensile strain modulates the homoepitaxial growth of $\mathrm{WSe}_{2}$ bilayer, we applied a two-step growth procedure where a monolayer $\mathrm{WSe}_{2}$ is grown on a sapphire followed by the van der Waals epitaxy normal to the basal plane growth front. The ability to systematically manipulate the built-in strain fields enables us to prepare the $\mathrm{WSe}_{2}$ monolayers with various tensile strain levels as the templates to direct the growth of the subsequent second $\mathrm{WSe}_{2}$ overlayer. As summarized in Figure $2 \mathrm{a}$, the built-in strain fields of the first epilayer $\mathrm{WSe}_{2}$ indeed play an important role in modulating the epitaxial growth front for the formation of $\mathrm{WSe}_{2}$ homobilayers. The $\mathrm{HTS}-\mathrm{WSe} \mathrm{e}_{2}$ monolayer enables the layer-by-layer growth mode, whereas the LTS-WSe ${ }_{2}$ directs the island growth mode. Figure $2 b, e$ features the optical microscope (OM) images of the first epilayer of HTS- and LTS-WSe ${ }_{2}$, respectively. PL and Raman measurements were used to confirm the strain uniformity and were found to follow the similar trend (see the Supporting Information, Figure S7). Here, the first HTS-WSe $e_{2}$ monolayer template, even with the built-in strain of only $1.37 \%$, evidentially and sufficiently enables the layer-by-layer growth 

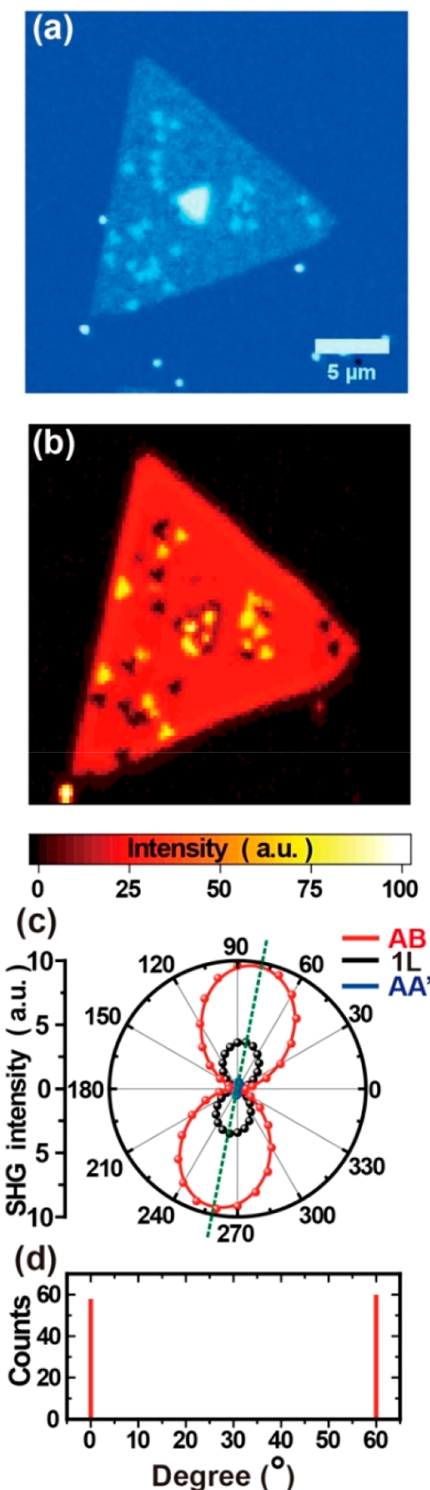
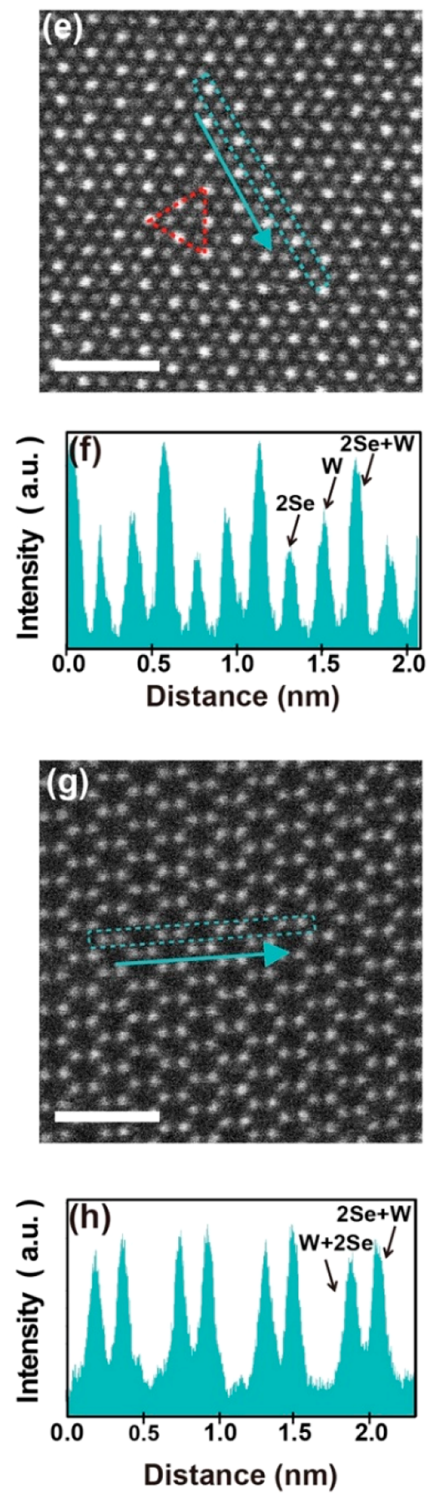

(i)

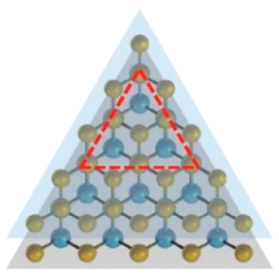

(j)
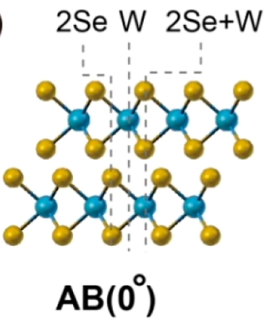

(k)

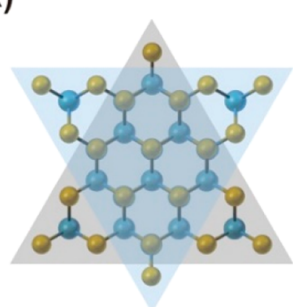

(I)

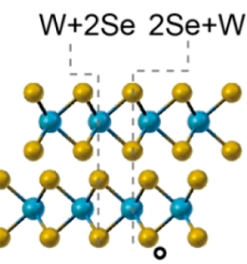

AA' $(60)$

Figure 3. Stacking configuration of $\mathrm{WSe}_{2}$ homobilayers. (a) The OM image shows the inception of triangular second WSe $\mathrm{e}_{2}$ overlayers grown on the first HTS-WSe $e_{2}$ epilayer. (b) The corresponding false-colored second harmonic intensity obtained by pixel-to-pixel spatial mappings in (a). (c) Polar plots of the polarization-resolved second harmonic intensity as a function of azimuthal angle $\varphi$ measured from an as-grown sample. (d) Statistical histograms of the twist angles measured from hundreds of the triangular second WSe $_{2}$ overlayers. (e) HAADF-STEM image of $\mathrm{WSe}_{2}$ homobilayers with a $0^{\circ}$ twist angle. (f) Intensity profile along the direction of selected line in (e). (g) HAADF-STEM image of $\mathrm{WSe}_{2}$ homobilayers with a $60^{\circ}$ twist angle. (h) Intensity profile along the direction of selected line in (g). Atomic configuration of $\mathrm{AB}\left(0^{\circ}\right.$ twisted, 3R) stacked $\mathrm{WSe}_{2}$ homobilayer, top view (i) and cross-sectional view (j). Atomic configuration of $\mathrm{AA}^{\prime}\left(60^{\circ}\right.$ twisted, $\left.2 \mathrm{H}\right)$ stacked $\mathrm{WSe}_{2}$ homobilayers, view from the top $(\mathrm{k})$ and the side (1). Blue balls denote $\mathrm{W}$ atoms, and yellow balls represent Se atoms (i-1). Scale bar: $1 \mathrm{~nm}$.

mode, whereas the epitaxy on LTS-WSe ${ }_{2}$ (a built-in strain of $0.56 \%)$ favors the island growth mode.

After the second growth, a uniform and fully covered second overlayer of $\mathrm{WSe}_{2}$ is epitaxially grown on top of the HTS-WSe epilayer as highlighted by the dashed triangle in red in Figure 2 c. The atomic force microscopy (AFM) image in Figure $2 \mathrm{~d}$ provides the close-up view that the growth proceeds with the seeding of small triangular $\mathrm{WSe}_{2}$ and then merges into a complete $\mathrm{WSe}_{2}$ bilayer. The height profile determined by a blue dashed line across the interface between a substrate and the first epilayer of $\mathrm{WSe}_{2}$ flakes is $0.82 \mathrm{~nm}$, and the second layer is $\sim 0.62 \mathrm{~nm}$, in accord with the signature thickness of monolayer $\mathrm{WSe}_{2}{ }^{18}$ Statistically, after scouring over 1000 flakes from various epitaxy batches, all of the $\mathrm{HTS}-\mathrm{WSe}_{2}$ templates proceed with the layer-by-layer growth mode without exception. Figure S8 showcases the low-magnification OM image where $\mathrm{WSe}_{2}$ homobilayers with uniform coverage are ubiquitous on all HTS-WSe $e_{2}$ epilayers. To the contrary, sporadically distributed islands or multilayer clusters (thickness up to $\sim 10 \mathrm{~nm}$ ) were found to scatter all over the LTS-WSe flakes as shown in Figure 2f,g. Note that, although the activation of the basal plane prioritizes the vertical growth front, the underlying $\mathrm{HTS}_{\mathrm{WSe}} \mathrm{W}_{2}$ templates concurrently become discernably larger. The dashed triangles in Figure $2 c, f$ present the size of the first epilayer in Figure $2 b, e$. Here, the edges of the first-layer $\mathrm{WSe}_{2}$ serve as the nucleation sites for the second in-plane growth of $\mathrm{WSe}_{2}$ during the ramping process. $^{35,36}$ To ensure the yield and coverage of the second 
(a)

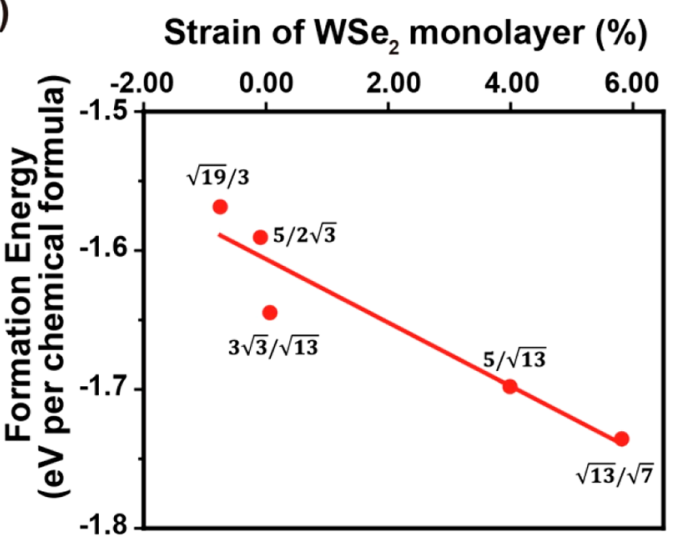

(c)

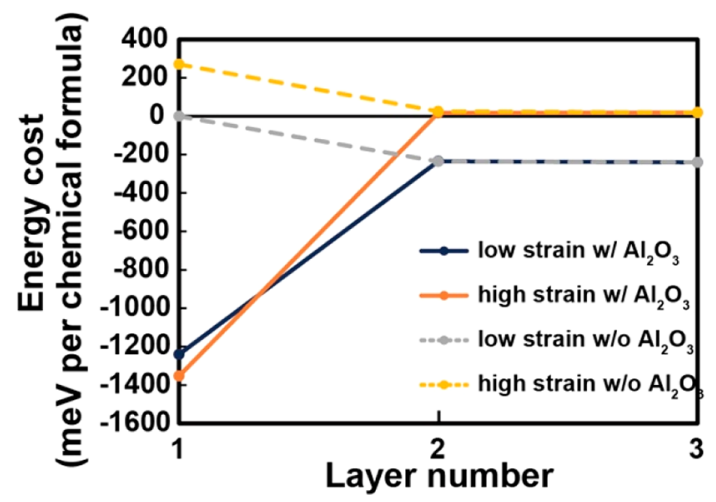

(b)

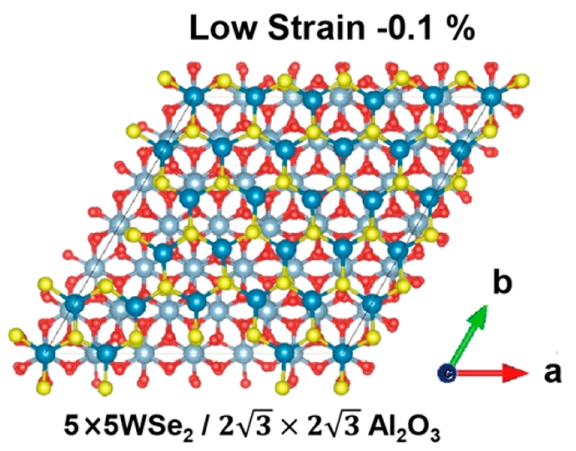

High Strain $5.8 \%$

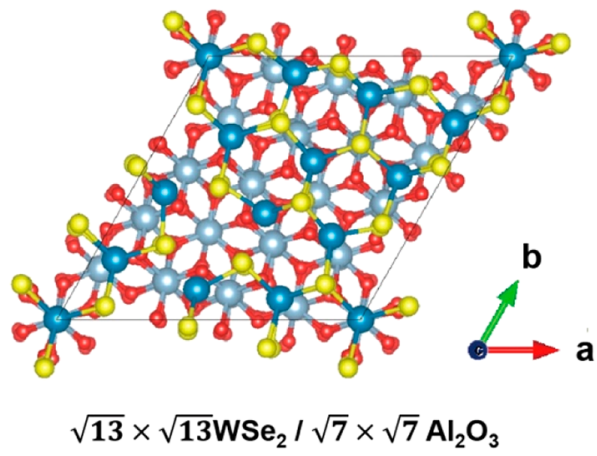

Figure 4. (a) Formation energy of monolayer $\mathrm{WSe}_{2}$ on sapphire $\left(\mathrm{Al}_{2} \mathrm{O}_{3}\right)$ superstructures with various strains. (b) Atomic models for the lowstrained $\left(5 \times 5 \mathrm{WSe}_{2} / 2 \sqrt{3} \times 2 \sqrt{3} \mathrm{Al}_{2} \mathrm{O}_{3}\right)$ and high-strained $\left(\sqrt{13} \times \sqrt{13} \mathrm{WSe}_{2} / \sqrt{7} \times \sqrt{7} \mathrm{Al}_{2} \mathrm{O}_{3}\right) \mathrm{WSe}_{2}$ on $\mathrm{Al}_{2} \mathrm{O}_{3}$. (c) The calculated energy cost of the $n$th layer WSe ${ }_{2}$ (with respect to the free-standing monolayer $\mathrm{WSe}_{2}$ ) on two systems with substrates, defined by $E_{n}=E_{(n) \mathrm{WS} e_{2} / \mathrm{Al}_{2} \mathrm{O}_{3}}-$ $E_{(n-1) \mathrm{WSe}_{2} / \mathrm{Al}_{2} \mathrm{O}_{3}}-E_{\text {free-WSe }}$ and other two without substrates.

$\mathrm{WSe}_{2}$ overlayer, the epitaxy growth should be conducted in the high-temperature region $\left(\sim 900{ }^{\circ} \mathrm{C}\right)$ where adequate energy overcomes the nucleation barrier on the basal plane of the HTS-WSe $e_{2}$ templates, therefore proceeding with the thermodynamically favorable stacking structure afterward. ${ }^{12,14}$ An epitaxial growth at the lower temperature leads to edgedirected in-plane growth (see Figure S9) and ultimately extends to the continuous $\mathrm{WSe}_{2}$ thin films. Figure $\mathrm{S} 10$ shows a typical distribution of second-growth results of $\mathrm{HTS}_{-\mathrm{WSe}}$ epilayers. At the very front end, where the HTS-WSe ${ }_{2}$ sample is placed right next to the $\mathrm{W}$ source, continuous multilayers (three to four layers) were developed on account of the sample precursor supply. The number of layers sequentially decreases to two to three layers and is ultimately limited to only bilayers when the HTS-WSe $e_{2}$ sample gradually moves away from the W source. The same trend was also observed in the case of a lowpressure CVD monolayer growth. ${ }^{37}$ It is noted that the scattered bright spots are thick nucleation sites. The presence of thick seeds can be suppressed efficiently by increasing the Se vapor flux or decreasing the $\mathrm{H}_{2} / \mathrm{Ar}$ ratio to restrain the $\mathrm{W}$ supply during the second growth.

Characterization of as-grown multilayer $\mathrm{WSe}_{2}$. The successful activation of the basal plane through the strain engineering of the first $\mathrm{WSe}_{2}$ epilayer guides the layer-by-layer growth to obtain a bilayer and even the trilayer as shown in Figure S11. AFM images reveal that the triangular $\mathrm{WSe}_{2}$ seeds the first nucleate, merges with the adjacent flakes, and ultimately forms a complete film prior to the growth of subsequent layers, closely resembling the layer-by-layer growth mode. Aside from the consistently aligned vertical growth on a basal plane, the first HTS-WSe $e_{2}$ templates and the second $\mathrm{WSe}_{2}$ overlayers are highly aligned, and the dominant twist angles/edge orientations are $0^{\circ}$ and $60^{\circ}\left(180^{\circ}\right)$. To verify the orientation of individual flakes, the $\mathrm{WSe}_{2}$ homobilayers were characterized by second-harmonic generation (SHG). It is known that polarization-resolved SHG is very sensitive to the crystal orientation and that the intensity profile map can be used as a descriptor for verifying spatial orientations between the first $\mathrm{HTS}_{\mathrm{W}} \mathrm{WS} \mathrm{e}_{2}$ epilayer and the second $\mathrm{WSe}_{2}$ overlayer. With this in mind, we specifically selected $\mathrm{WSe}_{2}$ homobilayers with incomplete coverage of second $\mathrm{WSe}_{2}$ overlayers as shown in Figure S11a to further enhance the fidelity of grain identification. Figure $3 a, b$ shows the OM image and the corresponding SHG intensity map of the triangular second $\mathrm{WSe}_{2}$ overlayers, which is independent of the polarization direction of the incident laser field with respect to the crystallographic axis. Here, the total SHG intensity of the sample was recorded. It is widely recognized that materials with the absence of inversion symmetry give rise to the SHG signal. Here, monolayer $\mathrm{WSe}_{2}$ is characterized by the $D_{3 h}$ point-group symmetry, which enables a strong SHG signal by virtue of its broken inversion symmetry. As a result, in the case of $\mathrm{WSe}_{2}$ homobilayers, the 
enhanced SHG intensity plot (bright) that stemmed from a parallel stacking $\left(0^{\circ}\right)$ is the result of $3 \mathrm{R}$ stacking or $\mathrm{AB}$ stacking, which can be assigned to the $D_{3 h}$ point group with broken inversion symmetry. On the contrary, the muchsuppressed second harmonic intensity plot (dark) taken at an antiparallel stacking $\left(60^{\circ}\right)$ is the consequence of the Bernal $(2 \mathrm{H})$ stacking or $\mathrm{AA}^{\prime}$ stacking (the $D_{3 d}$ point group) with inversion symmetry. ${ }^{38}$ We further examined the polarizationresolved SHG as shown in Figure 3c. The polarization direction for bilayer homostructures with different twist angles seems to align well with the first HTS-WSe $\mathrm{H}_{2}$ templates, an indication of the formation of energetically and thermodynamically favorable stacking between the second overlayers on the first HTS-WSe ${ }_{2}\left(0^{\circ}\right.$ or $\left.60^{\circ}\right)$. In addition, we extended the SHG measurements to different batches of $\mathrm{WSe}_{2}$ homobilayers. From analyzing the compiled statistics shown in Figure $3 \mathrm{~d}$, it becomes clear that the stacking mode only shows two directions $\left(0^{\circ}\right.$ twist or $60^{\circ}$ twist $)$ that are generally recognized as the most stable stacking in $\mathrm{WSe}_{2}$ system. ${ }^{15,39}$ These results are consistent with the previous observation on artificially stacked bilayer $\mathrm{MoS}_{2}{ }^{38}$

In parallel, the stacking configurations of the $\mathrm{WSe}_{2}$ homobilayers were investigated by the plane-view scanning transmission electron microscopy (STEM) with the high-angle annular dark-field (HAADF) mode. This is because the $\mathrm{Z}$ contrast is highly dependent on the atomic numbers, making it possible to infer the detailed atomic arrangement. Here, $\mathrm{WSe}_{2}$ homobilayers comprised of the opposite twist angle $\left(0^{\circ}\right.$ and $60^{\circ}$ ) were selected (Figure S12a). Figure 3e,g features the high-resolution HAADF-STEM images taken at $\mathrm{WSe}_{2}$ homobilayers of $0^{\circ}$ and $60^{\circ}$ twist angles. Distinctively different atomic patterns become discernible as a result of different stacking sequences as shown in Figure S12b,c. In the $A B$ stacking configuration (twist angle of $0^{\circ}$, Figure $3 \mathrm{i}$ ), atoms intersected by the red dashed line represent the $2 \mathrm{Se}+\mathrm{W}$ overlaps. The bright spots marked in Figure $3 \mathrm{e}$ manifest the overlaps by means of sandwiching $\mathrm{W}$ atoms with two Se atoms in the vertical and aligned fashion. Furthermore, from the sectional view of the $\mathrm{WSe}_{2}$ bilayer stacking, it becomes apparent that the upper layer slides and thus gives rise to three atomic alignments, including $2 \mathrm{Se}, \mathrm{W}$, and $2 \mathrm{Se}+\mathrm{W}$ (Figure $3 \mathrm{j}$ ). The intensity profile extracted from the selected regions in Figure $3 \mathrm{e}$ also follows the similar trend in terms of stacking sequence as shown in Figure $3 \mathrm{f}$. Alternatively, the atomic configuration featured in Figure $3 \mathrm{k}$ with a $60^{\circ}$ rotation of the hexagonal structure results in the alignment of $\mathrm{W}$ with $\mathrm{Se}$, that is, the $\mathrm{AA}^{\prime}$ stacking with the atomic alignment of $\mathrm{W}+2 \mathrm{Se}$ and $2 \mathrm{Se}+\mathrm{W}$ (Figure 31 ), respectively. The intensity profile (Figure $3 \mathrm{~h}$ ) derived from Figure $3 \mathrm{~g}$ matches well with the aforementioned atomic stacking sequence. ${ }^{40}$ Moreover, the optical properties of as-grown $\mathrm{WSe}_{2}$ with different layer numbers are systematically investigated (Figure S13). The results suggest that the growth front through activating the basal plane during the epitaxy has made possible the preservation of all these characteristics unique to the exfoliated benchmarks, thus distinguishing the strain-engineering approach from the other strategies. ${ }^{38}$

Mechanism of strain-induced bilayer $\mathrm{WSe}_{2}$. To understand the correlation between strain and the associated growth modes, DFT computations were performed to probe the information on formation energy in the presence of strain. To this end, atomic models of monolayer $\mathrm{WSe}_{2}$ epitaxially grown on sapphire with various degrees of the magnitude of built-in strain ranging from $-1 \%$ to $6 \%$ were constructed. The formation energy is calculated for each superstructure that sits at the lowest energy among various atomic registries by sliding the layer surface with respect to the substrate. The calculated formation energy on the basal plane, $E_{\mathrm{f}}=E_{\mathrm{WS}_{2} / \mathrm{Al}_{2} \mathrm{O}_{3}}$ - $E_{\mathrm{WSe}_{2}}-E_{\mathrm{Al}_{2} \mathrm{O}_{3}}$, is found to decrease linearly as strain increases (Figure 4a), suggesting that the high tensile strain indeed arises from the strong interaction between the $\mathrm{WSe}_{2}$ and sapphire substrate. The formation energy $E_{\mathrm{f}}$ is known to be associated with the change of Gibbs free energy per unit volume of the solid phase, $\Delta G_{v}$. The change of volume of Gibbs free energy can be expressed as $\Delta G^{*}=\left(\frac{16 \pi \gamma V f}{3\left(\Delta G_{\mathrm{v}}+w\right)^{2}}\right)$ $\times\left(\frac{2-3 \cos \theta+\cos ^{3} \theta}{4}\right)$ where $\gamma$ is the surface tension, $\theta$ represents the contact angle, and $w$ denotes the strain energy per unit volume generated by the stress in the epitaxy. When the strain energy per unit volume generated by the disproportional concentration profile of $\mathrm{WO}_{3} /$ Se precursor vapors is included, the overall energy barrier to nucleation increases, because the sign of $\Delta G_{v}$ is negative, and the sign of strain energy is positive.

With this in mind, as shown in Figure $4 b$, we investigate the energetics of the layer-by-layer growth mode of the subsequent second $\mathrm{WSe}_{2}$ overlayer. Two atomic models for the LTS $(5 \times$ $\left.5 \mathrm{WSe}_{2} / 2 \sqrt{3} \times 2 \sqrt{3} \mathrm{Al}_{2} \mathrm{O}_{3}\right)$ and HTS $\left(\sqrt{13} \times \sqrt{13} \mathrm{WSe}_{2} /\right.$ $\left.\sqrt{7} \times \sqrt{7} \mathrm{Al}_{2} \mathrm{O}_{3}\right) \mathrm{WSe}_{2}$ on sapphire corresponds to the two extremities of the $\mathrm{WSe}_{2}$-sapphire interactions: (i) nearly strainfree $(-0.1 \%)$ and (ii) extremely high orders of magnitude of strain level of $5.8 \%$, respectively. Figure $4 \mathrm{c}$ shows the calculated energy cost of the formation of the $n$th $(n=$ $1,2,3)_{\mathrm{WSe}_{2}}$ overlayer (with regard to free-standing single layer) on the two aforementioned systems, defined by $E_{n}=$ $E_{(n) \mathrm{WSe}_{2} / \mathrm{Al}_{2} \mathrm{O}_{3}}-E_{(n-1) \mathrm{WSe}_{2} / \mathrm{Al}_{2} \mathrm{O}_{3}}-E_{\text {free-WSe }}$. Another two reference cases, HTS-WSe $e_{2}$ and LTS-WSe $e_{2}$ in a freestanding form, that is, without the supporting $\mathrm{Al}_{2} \mathrm{O}_{3}$ substrates, are also included for comparison. It is found that the energetic landscape of the subsequent epilayer always shifts toward the lower end in the less-strained system (the difference is $260-$ $270 \mathrm{meV}$ per chemical formula for $n=2$ and 3). Meanwhile, after including the underlying $\mathrm{Al}_{2} \mathrm{O}_{3}$ growth substrates, the energy cost of the first $\mathrm{WSe}_{2}$ epilayer for the high tensile strain case becomes even lower than that of a low-strain case, shifting by $112 \mathrm{meV}$ per chemical formula. This shift in energy cost thus suggests the presence of a strong interaction between the first $\mathrm{WSe}_{2}$ epilayer and $\mathrm{Al}_{2} \mathrm{O}_{3}$ in the high tensile strain cases. (Note that the distance between the bottom Se layer and the O-termination surface is 1.77 and $1.7 \AA$ for low- and highstrain systems.) The energy cost for adding second and third $\mathrm{WSe}_{2}$ overlayers becomes nearly identical with or without substrates (Figure 4). In parallel, contributions to the layer-bylayer energy cost of the $\mathrm{WSe}_{2} / \mathrm{Al}_{2} \mathrm{O}_{3}$ system are twofold: substrate interaction and strain effect, with the former being negative, and the latter being positive. In the stronger interaction with an underlying substrate in a $\mathrm{HTS}_{\mathrm{WSe}}$ induced Frank-van der Merwe (layer-by-layer) growth mode, ${ }^{41}$ adatoms attach preferentially to surface sites resulting in a laterally grown film prior to the growth of subsequent layers along the vertical direction. Antithetically, a negligible strain in LTS-WSe ${ }_{2}$ leads to a Volmer-Weber (nucleation-toisland) growth, ${ }^{42}$ indicating a very weak interaction with the surface, such that adatom-adatom interactions are stronger 
(a)

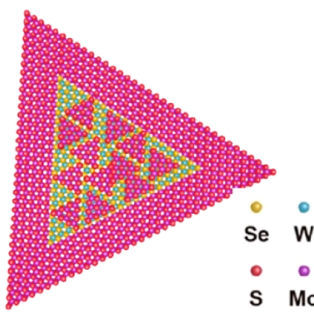

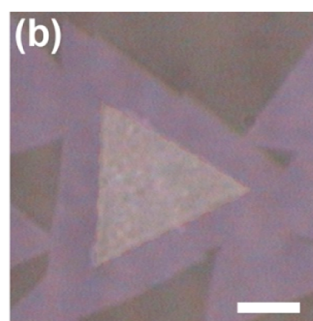

(c)

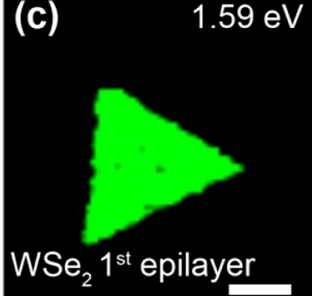

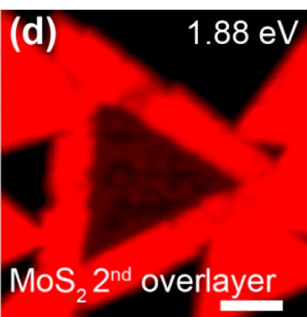

(e)

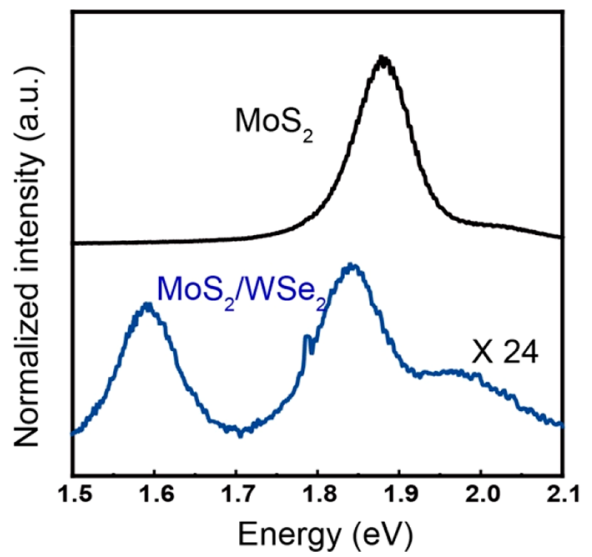

(f)

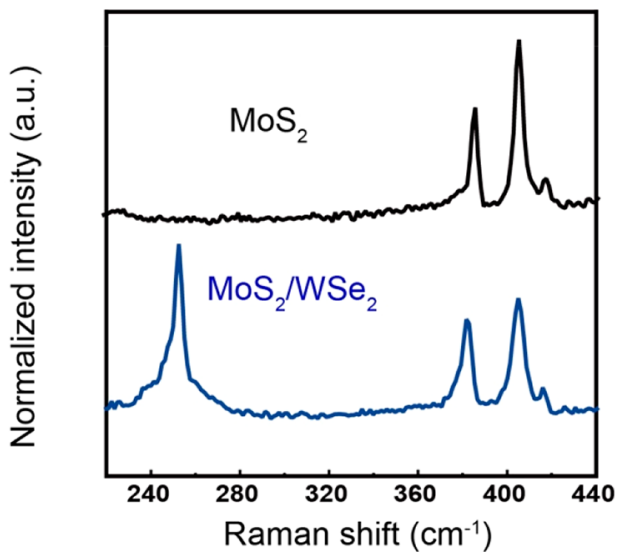

Figure 5. $\mathrm{MoS}_{2} / \mathrm{WSe}_{2}$ heterostructure obtained from the first HTS-WSe $\mathrm{H}_{2}$. (a) Schematic illustration of atomic structures and (b) OM image of vertical and lateral $\mathrm{MoS}_{2} / \mathrm{WSe}_{2}$ heterostructures. (c, d) The corresponding PL mapping images at 1.59 and $1.88 \mathrm{eV}$, respectively. (e) PL spectra and (f) Raman spectra corrected from the core (blue) and surrounding (black) regions. Scale bars: $5 \mu \mathrm{m}$.

than those of the adatom with the surface, resulting in the vertical growth with adatom clusters or islands. As a result, the layer-by-layer vertical growth of WSe $e_{2}$ bilayers is energetically preferred at the beginning of the nucleation stage and becomes thermodynamically stable at the end of the epitaxial growth by virtue of compensating the energy cost induced by the high tensile strain $\left(E_{2,3}>0\right)$. For low-strain (nearly strain-free) systems, the total energy cost is always negative, and therefore the growth of a subsequent second $\mathrm{WSe}_{2}$ overlayer favors the nucleation-to-island mode. Our experimental observation and simulations agree well with the above thin-film growth models. Growth and Characterization of $\mathrm{MoS}_{2} / \mathrm{WSe}_{2}$ Heterobilayers. Because our strain-engineering epitaxy is directed by the builtin strain fields, intrinsic to the 2D TMDC growth, its use is not limited to the $\mathrm{WSe}_{2}-\mathrm{WSe}_{2}$ homobilayers reported here. Instead, it could be generalized for epitaxy growth of vdW heterobilayers with atomically thin $\mathrm{p}-\mathrm{n}$ junctions. Here, combined with the understanding of the nucleation and growth characteristics on an $\mathrm{HTS}-\mathrm{WSe}_{2}$ template, we expanded the strain-induced epitaxy growth to include $\mathrm{MoS}_{2} / \mathrm{WSe}_{2} \mathrm{p}-\mathrm{n}$ heterobilayers. To this end, HTS-WSe ${ }_{2}$ first epilayers were again featured as the strained template for the subsequent growth of second $\mathrm{MoS}_{2}$ overlayers. Note that a two-step growth in different furnaces is used to afford the heterostructures without cross contamination at the atomically thin $\mathrm{p}-\mathrm{n}$ interfaces. The growth temperature of second $\mathrm{MoS}_{2}$ overlayers is set at $750-760{ }^{\circ} \mathrm{C}$ under 30 Torr with $\mathrm{Ar}$ as the carrier gas. Figure 5 a schematically illustrates the sequential epitaxy growth of second $\mathrm{MoS}_{2}$ overlayers. Both vertical and lateral epitaxy growths of $\mathrm{MoS}_{2}$ on the HTS-WSe templates are observed with the vertical growth front (basal plane) outweighing the lateral counterparts (edges). This can be understood from the epitaxial point of view that the formation of lateral $\mathrm{p}-\mathrm{n}$ junctions takes place during the ramping of annealing temperatures for the second growth. ${ }^{35,43}$ We specifically selected $\mathrm{WSe}_{2} / \mathrm{MoS}_{2}$ heterobilayers with an incomplete coverage of a second $\mathrm{MoS}_{2}$ epilayer as a result of PL quenching, which can be leveraged as a descriptor to identify the location of second $\mathrm{MoS}_{2}$ epilayers. Figure $5 \mathrm{~b}$ shows the OM image of the as-grown $\mathrm{MoS}_{2} / \mathrm{WSe}_{2}$ heterobilayers. The distribution of the first $\mathrm{WSe}_{2}$ template and the second $\mathrm{MoS}_{2}$ overlayer was further examined by PL intensity maps at $1.59 \mathrm{eV}$ (wavelength of $780 \mathrm{~nm}$ ) and $1.88 \mathrm{eV}$ (wavelength of $660 \mathrm{~nm}$ ), characteristic of the direct excitonic transitions in both $\mathrm{MoS}_{2}$ and $\mathrm{WSe}_{2}$. A PL mapping of relevant PL characteristics, including $\mathrm{MoS}_{2}$ in green (Figure 5c) and $\mathrm{WSe}_{2}$ in red (Figure $5 \mathrm{~d}$ ), evidentially proves the successful outof-plane growth of n-type $\mathrm{MoS}_{2}$ on the basal plane of p-type $\mathrm{WSe}_{2}$. In addition, the PL spectra in Figure 5e reveal diverse PL emission peaks at the core (blue) and from the surrounding (black) regions. The black curve with a prominent emission peak at $1.88 \mathrm{eV}$ represents the direct bandgap excitonic peak of monolayer $\mathrm{MoS}_{2}$. Meanwhile, the emergence of two relatively weak peaks in blue $(1.59 \mathrm{vs} 1.85 \mathrm{eV})$ was observed in the core region. The peak assigned at $1.59 \mathrm{eV}$ corresponds to the combination of intralayer excitonic emission from the underlying $\mathrm{WSe}_{2}$ epilayer $\left(\mathrm{X}_{\mathrm{WSe} 2}\right.$ at $\left.1.61 \mathrm{eV}\right)$ and the $\mathrm{MoS}_{2} /$ $\mathrm{WSe}_{2}$ interlayer exiton $\left(\mathrm{X}_{\mathrm{MoS} 2 / \mathrm{WSe} 2}\right.$ at $\left.1.58 \mathrm{eV}\right)$, as shown in Figure S14. ${ }^{44}$ The considerable deviation and intensity quenching of PL emission can be translated into the strong interlayer coupling and charge transfer, characteristic of the type II heterostructures. ${ }^{45}$ Similarly, Raman spectra acquired 
from the core region (black) and surrounding (blue) area of the $\mathrm{MoS}_{2} / \mathrm{WSe}_{2}$ heterostructures were summarized in Figure 5f. Two predominant peaks at 405 and $385 \mathrm{~cm}^{-1}$, which correspond to the $\mathrm{A}_{1 \mathrm{~g}}$ and $\mathrm{E}_{2 \mathrm{~g}}$ modes of monolayer $\mathrm{MoS}_{2}$, arise from the surrounding. Nevertheless, the core region exhibits both characteristic peaks of $\mathrm{MoS}_{2}$ and $\mathrm{WSe}_{2}\left(250 \mathrm{~cm}^{-1}, \mathrm{E}_{2 \mathrm{~g}}\right.$ mode), respectively. The presence of relevant Raman characteristics in tandem with representative features from both PL spectra and mappings collectively manifests the applicability and generality of a strain-induced epitaxy growth of heterobilayers with atomically thin and clean $p-n$ interfaces. A wide variety of artificial TMDC heterostackings with control over the number of layers, uniformity, coverage, and intrinsic material properties can thus be epitaxially grown for a myriad of electronic and optoelectronic applications.

Conclusions. In conclusion, we have demonstrated that built-in strain fields in 2D TMDCs play a crucial role in activating the out-of-plane growth front for affording homo- and heterobilayers. With the consistent agreement between DFT simulations, experimental observations, and spectroscopic characterizations, our findings elucidate general rules for a strain-driven epitaxy growth of homo- and heterobilayers: (1) the overall energy barrier to rampant nucleation increases because of the presence of strain energy that reconciles the adverse impact of $\Delta G_{\mathrm{v}}$. The result is the activation of the growth front on the basal plane where epitaxy of bilayer proceeds, (2) built-in strain fields in the first $\mathrm{WSe}_{2}$ epilayers stem from the combined effects of local chemical environments and can be systematically modulated in situ during the epitaxy growth, (3) the stacking of homo- and heteroepilayers predominately follows $\mathrm{AB}\left(0^{\circ}\right.$ twisted, 3R) and $\mathrm{AA}^{\prime}\left(60^{\circ}\right.$ twisted, $2 \mathrm{H}$ ) according to the analyses of SHG measurement and HAADF-STEM images, and (4) this strain-modulated epitaxy can be a general platform for extending libraries of vdW homo- and heterobilayers. Such an understanding not only provides fundamental insights into the knowledge for the vapor-phase epitaxy of $2 \mathrm{D}$ layers but opens new inroads in the direct growth of vdW homo- and heterobilayers with control over the bilayer coverage and content in tandem with the preservation of electrically addressable and spectroscopically tractable interfaces in a potentially scalable and industrycompatible fashion.

Materials and Methods

Growth of monolayer, WSe $\mathrm{W}_{2}$ homobilayers, and $\mathrm{MoS}_{2} / \mathrm{WSe}_{2}$ heterobilayers. First, the epitaxy growth of single-crystal first $\mathrm{WSe}_{2}$ epilayers was adopted from the previous work. ${ }^{18}$ In essence, $\mathrm{WO}_{3}$ (Sigma-Aldrich, 99.9\%) powders were placed in a ceramic boat that was located at the center of the furnace. The sapphire substrates were located in the downstream side adjacent to the ceramic boat as shown in Figure 1. The Se powders (Sigma-Aldrich, 99.99\%) were annealed by a heating tape at the upstream side, and the resulting Se vapors were transported by an $\mathrm{Ar} /$ hydrogen $\left(\mathrm{H}_{2}\right)$ mixture gas (66 to 5 $\mathrm{sccm})$. The furnace was then heated to $900{ }^{\circ} \mathrm{C}$ with a heating rate of $25{ }^{\circ} \mathrm{C} / \mathrm{min}$ at 10 Torr. The duration of the epitaxy growth of the first $\mathrm{WSe}_{2}$ was $\sim 15 \mathrm{~min}$, subjected to the variation in ramping rate and loading of precursors. Upon completion, the system was naturally cooled to room temperature. The production of both HTS-WSe $e_{2}$ and LTS$\mathrm{WSe}_{2}$ was accomplished by adjusting the precursor ratio between $\mathrm{W}$ and Se. For obtaining the WSe $\mathrm{W}_{2}$ homobilayers, the resultant $\mathrm{HTS}-\mathrm{WSe}_{2}$ along with the sapphire substrate was directly used as the template and was placed in the downstream side. Note that the growth of a second epilayer was performed under a Se-rich environment. Through control of the growth time, a fully covered $\mathrm{WSe}_{2}$ homobilayer film can be achieved. In the case of synthesizing the $\mathrm{MoS}_{2} / \mathrm{WSe}_{2}$ heterobilayers, the as-grown $\mathrm{HTS}_{\mathrm{WSe}}$ flakes/films were transferred into another CVD furnace to avoid crosscontamination. The furnace temperature was set as $800{ }^{\circ} \mathrm{C}$ with the surface temperature of growth substrate measured at $\sim 750-760{ }^{\circ} \mathrm{C}$, and the duration of the epitaxy growth lasted for $10 \mathrm{~min}$. Importantly, in the $\mathrm{MoS}_{2}$ furnace, $S$ powders (Sigma-Aldrich, 99.9\%) and $\mathrm{MoO}_{3}$ powders (Sigma-Aldrich, 99.9\%) were used as precursors. The flow of carrier gas (Ar) was carefully maintained at $70 \mathrm{sccm}$, and the pressure was controlled at 30 Torr.

Raman \& photoluminescence characterization. The Raman and photoluminescence (PL) spectra on $\mathrm{WSe}_{2}$ homobilayers and $\mathrm{WSe}_{2} / \mathrm{MoS}_{2}$ heterobilayers were collected using a Witec alpha 300 confocal Raman microscope equipped with a RayShield coupler. A $532 \mathrm{~nm}$ solid-state laser was the excitation source. The excitation light with a power of 2.5 $\mathrm{mW}$ was focused onto the sample by a $100 \times$ objective lens (numerical aperture $(\mathrm{NA})=0.9)$. The signal was collected by the same objective lens, analyzed by a $0.75 \mathrm{~m}$ monochromator, and detected by a liquid-nitrogen-cooled charge-coupled device (CCD) camera. The atomic force microscopy (Cypher ES-Asylum Research Oxford Instruments) characterizations were conducted with Olympus (OMCLAC240TS) Al-coated silicon cantilevers. The resonance frequency was $\sim 70 \mathrm{kHz}$, the spring constant was $\sim 2 \mathrm{~N} / \mathrm{m}$, and the tip curvature radius was $\sim 7 \mathrm{~nm}$. The temperature-dependent PL spectra on monolayer HTS-WSe $_{2}$ and LTS-WSe ${ }_{2}$ flakes was measured by a microphotoluminescence setup integrated in a cryogen-free cryostat with a base temperature of $4.2 \mathrm{~K}$. A He-Ne laser with a wavelength of $632.8 \mathrm{~nm}$ was focused by a $100 \times$ objective lens $(\mathrm{NA}=0.82)$ to excite the $\mathrm{WSe}_{2}$ flakes. The PL signals were collected by the same objective lens and sent to a liquidnitrogen-cooled CCD detector.

Second-harmonic generation measurements. SHG spectroscopy was conducted by a backscattering optical microscope at room temperature. ${ }^{38}$ The fundamental field was provided by a mode-locked Ti:sapphire pulsed laser $(870 \mathrm{~nm})$ and focused on the sample surface by a $100 \times$ objective lens $(\mathrm{NA}=0.9)$. The backscattered SHG signals were collected by the same objective lens, analyzed by a $0.75 \mathrm{~m}$ monochromator, and detected by a liquid-nitrogen-cooled CCD camera. The polarization of the fundamental laser (SHG signal) was selected (analyzed) by combining a linear polarizer and a half-wave plate. For spatial SHG mappings, the sample was mounted on a motorized $x-y$ scanning stage with the high repeatability of $0.25 \mu \mathrm{m}$.

Scanning transmission electron microscopy. The homo- and heterobilayer samples for STEM were prepared by wet-transfer methods. Specifically, poly(methyl methacrylate) (PMMA) was spin-coated onto the as-grown $\mathrm{WSe}_{2}$ homobilayer samples as a supporting substrate. The PMMA/WSe ${ }_{2}$ homobilayer composites were fully soaked into HF solution, leaving behind the $\mathrm{WSe}_{2}$ homobilayers. After a rinse with copious amounts of deionized water $\left(\mathrm{DI}-\mathrm{H}_{2} \mathrm{O}\right)$, suspended $\mathrm{WSe}_{2}$ homobilayers were scooped onto transmission electron microscopy (TEM) grids. To enhance the adhesion between $\mathrm{WSe}_{2}$ homobilayer samples and the underlying TEM grids, an additional annealing was performed at $50{ }^{\circ} \mathrm{C}$ for $1 \mathrm{~h}$. Then the residual PMMA was fully removed by a thorough acetone rinse. The HAADF- 
STEM imaging was conducted at $80 \mathrm{kV}$ using a JEOL ARM 200F transmission electron microscope $(80-200 \mathrm{kV})$. The TEM is equipped with a Cs (spherical aberration) corrector and a high brightness cold field-emission gun (C-FEG), and the STEM detector is made of yttrium aluminum perovskite (YAP).

Density function theory simulation. The first-principles calculations were performed with DFT as implemented in the Vienna $\mathrm{Ab}$ initio Simulation Package (VASP). ${ }^{46}$ The interaction between electrons and ionic cores was approximated by the projector augmented wave method, and the exchange-correlation potential was described by the PBEGGA, ${ }^{47}$ with the vdW correction vdW-DF (optB86) functionals. ${ }^{48}$ A slab model is used for simulation with a vacuum thickness larger than $18 \AA$ to eliminate the spurious interaction, and the plane waves energy cutoff is $400 \mathrm{eV}$. The structure was fully relaxed until the change of the energy and the force reached $10^{-5} \mathrm{eV}$ per $1 \times 1$ cell and $10^{-3} \mathrm{eV} / \AA$, respectively. A $2 \times 2 \times 1$ and $3 \times 3 \times 1$ k-grid was used for low-strained $\left(5 \times 5 \mathrm{WSe}_{2} / 2 \sqrt{3} \times 2 \sqrt{3} \quad \mathrm{Al}_{2} \mathrm{O}_{3}\right)$ and highstrained $\left(\sqrt{13} \times \sqrt{13} \mathrm{WSe}_{2} / \sqrt{7} \times \sqrt{7} \mathrm{Al}_{2} \mathrm{O}_{3}\right)$ systems, with an interlayer distance of 1.77 and $1.70 \AA$ between the $\mathrm{WSe}_{2}$ and $\mathrm{Al}_{2} \mathrm{O}_{3}$ and an interlayer distance between 6.58 and $6.5 \AA$ for $\mathrm{WSe}_{2}$ and $\mathrm{WSe}_{2}$. The lattice constants of $\mathrm{WSe}_{2}$ and sapphire used for calculations are 3.28 and $4.73 \AA$.

\section{ASSOCIATED CONTENT}

\section{(s) Supporting Information}

The Supporting Information is available free of charge at https://pubs.acs.org/doi/10.1021/acsmaterialslett.0c00554.

Plot of energy distribution, discussion of temperaturedependent PL spectra, SEM images, discussion of the modified growth process, discussion of mismatch in the thermal expansion coefficient, temperature variation along diffusion pathway, microscopy and spectroscopy characterizations, discussion of optical properties (PDF)

\section{AUTHOR INFORMATION}

\section{Corresponding Authors}

Vincent Tung - Physical Sciences and Engineering Division, King Abdullah University of Science and Technology, Thuwal 23955-6900, Kingdom of Saudi Arabia; (1) orcid.org/00000003-3230-0932; Email: vincent.tung@kaust.edu.sa

Lain-Jong Li - Physical Sciences and Engineering Division, King Abdullah University of Science and Technology, Thuwal 23955-6900, Kingdom of Saudi Arabia; School of Materials Science and Engineering, University of New South Wales, Sydney 2052, New South Wales, Australia; Email: lance.li@ kaust.edu.sa

\section{Authors}

Yi Wan - Physical Sciences and Engineering Division, King Abdullah University of Science and Technology, Thuwal 23955-6900, Kingdom of Saudi Arabia; 다. orcid.org/00000002-6202-4193

Jing-Kai Huang - School of Materials Science and Engineering, University of New South Wales, Sydney 2052, New South Wales, Australia

Chih-Piao Chuu - Institute of Atomic and Molecular Sciences, Academia Sinica, Taipei 11529, Taiwan

Wei-Ting Hsu - Department of Physics, National Tsing Hua University, Hsinchu 30010, Taiwan
Chien-Ju Lee - Department of Physics, National Tsing Hua University, Hsinchu 30010, Taiwan

Areej Aljarb - Physical Sciences and Engineering Division, King Abdullah University of Science and Technology, Thuwal 23955-6900, Kingdom of Saudi Arabia

Chun-Wei Huang - Material and Chemical Research Laboratories, Nanotechnology Research Center, Industrial Technology Research Institute, Hsinchu 310, Taiwan

Ming-Hui Chiu - Physical Sciences and Engineering Division, King Abdullah University of Science and Technology, Thuwal 23955-6900, Kingdom of Saudi Arabia; (1) orcid.org/00000003-3753-8149

Hao-Ling Tang - Physical Sciences and Engineering Division, King Abdullah University of Science and Technology, Thuwal 23955-6900, Kingdom of Saudi Arabia

Ci Lin - Physical Sciences and Engineering Division, King Abdullah University of Science and Technology, Thuwal 23955-6900, Kingdom of Saudi Arabia

Xuechun Zhang - Physical Sciences and Engineering Division, King Abdullah University of Science and Technology, Thuwal 23955-6900, Kingdom of Saudi Arabia

Ching-Ming Wei - Institute of Atomic and Molecular Sciences, Academia Sinica, Taipei 11529, Taiwan; 이잉.org/00000003-3984-4422

Sean Li - School of Materials Science and Engineering, University of New South Wales, Sydney 2052, New South Wales, Australia

Wen-Hao Chang - Department of Physics, National Tsing Hua University, Hsinchu 30010, Taiwan; 아이.org/ 0000-0003-4880-6006

Complete contact information is available at:

https://pubs.acs.org/10.1021/acsmaterialslett.0c00554

\section{Notes}

The authors declare no competing financial interest.

\section{ACKNOWLEDGMENTS}

V.T. and L.-J.L. acknowledge the support from King Abdullah University of Science and Technology (KAUST) Office of Sponsored Research (OSR) under Award No. OSR-2018CARF/CCF-3079. V.T. is grateful for the support from KAUST Catalysis Center. A.A. and M.-H.C. are supported by KAUST Solar Center. W.H.C. acknowledges the support from the Ministry of Science and Technology of Taiwan (MOST-108-2119-M-009-011-MY3, MOST-107-2112-M-009024-MY3) and from the CEFMS of NCTU supported by the Ministry of Education of Taiwan. V.T. and Y.W. are indebted to the support from Core Lab in KAUST and the fruitful discussions in DFT with Dr. Z. Cao.

\section{REFERENCES}

(1) Wen, W.; Wu, L.; Yu, T. Excitonic Lasers in Atomically Thin 2D Semiconductors. ACS Materials Letters 2020, 2 (10), 1328-1342.

(2) Yang, C.-W.; Tang, H.-L.; Sattar, S.; Chiu, M.-H.; Wan, Y.; Chen, C.-H.; Kong, J.; Huang, K.-W.; Li, L.-J.; Tung, V. Epitaxial Growth and Determination of Band Alignment of Bi2Te3-WSe2 Vertical van der Waals Heterojunctions. ACS Materials Letters 2020, 2 (10), 1351-1359.

(3) Novoselov, K. S.; Mishchenko, A.; Carvalho, A.; Castro Neto, A. H. 2D materials and van der Waals heterostructures. Science 2016, 353 (6298), aac9439.

(4) Lee, C.-H.; Lee, G.-H.; van der Zande, A. M.; Chen, W.; Li, Y.; Han, M.; Cui, X.; Arefe, G.; Nuckolls, C.; Heinz, T. F.; Guo, J.; Hone, 
J.; Kim, P. Atomically thin $\mathrm{p}-\mathrm{n}$ junctions with van der Waals heterointerfaces. Nat. Nanotechnol. 2014, 9 (9), 676-681.

(5) Howell, S. L.; Jariwala, D.; Wu, C.-C.; Chen, K.-S.; Sangwan, V. K.; Kang, J.; Marks, T. J.; Hersam, M. C.; Lauhon, L. J. Investigation of band-offsets at monolayer-multilayer MoS2 junctions by scanning photocurrent microscopy. Nano Lett. 2015, 15 (4), 2278-2284.

(6) Brar, V. W.; Sherrott, M. C.; Jariwala, D. Emerging photonic architectures in two-dimensional opto-electronics. Chem. Soc. Rev. 2018, 47 (17), 6824-6844.

(7) Natori, K. Ballistic metal-oxide-semiconductor field effect transistor. J. Appl. Phys. 1994, 76 (8), 4879-4890.

(8) Kim, S.; Konar, A.; Hwang, W.-S.; Lee, J. H.; Lee, J.; Yang, J.; Jung, C.; Kim, H.; Yoo, J.-B.; Choi, J.-Y.; Jin, Y. W.; Lee, S. Y.; Jena, D.; Choi, W.; Kim, K. High-mobility and low-power thin-film transistors based on multilayer MoS2 crystals. Nat. Commun. 2012, 3, 1011.

(9) Choi, W.; Cho, M. Y.; Konar, A.; Lee, J. H.; Cha, G.-B.; Hong, S. C.; Kim, S.; Kim, J.; Jena, D.; Joo, J.; Kim, S. High-Detectivity Multilayer MoS2 Phototransistors with Spectral Response from Ultraviolet to Infrared. Adv. Mater. 2012, 24 (43), 5832-5836.

(10) Lee, H. S.; Min, S.-W.; Chang, Y.-G.; Park, M. K.; Nam, T.; Kim, H.; Kim, J. H.; Ryu, S.; Im, S. MoS2 nanosheet phototransistors with thickness-modulated optical energy gap. Nano Lett. 2012, 12 (7), 3695-3700.

(11) Liu, K.; Yan, Q.; Chen, M.; Fan, W.; Sun, Y.; Suh, J.; Fu, D.; Lee, S.; Zhou, J.; Tongay, S.; Ji, J.; Neaton, J. B.; Wu, J. Elastic Properties of Chemical-Vapor-Deposited Monolayer MoS2, WS2, and Their Bilayer Heterostructures. Nano Lett. 2014, 14 (9), 5097-5103.

(12) Ye, H.; Zhou, J.; Er, D.; Price, C. C.; Yu, Z.; Liu, Y.; Lowengrub, J.; Lou, J.; Liu, Z.; Shenoy, V. B. Toward a Mechanistic Understanding of Vertical Growth of van der Waals Stacked 2D Materials: A Multiscale Model and Experiments. ACS Nano 2017, 11 (12), 12780-12788.

(13) Li, F.; Feng, Y.; Li, Z.; Ma, C.; Qu, J.; Wu, X.; Li, D.; Zhang, X.; Yang, T.; He, Y.; Li, H.; Hu, X.; Fan, P.; Chen, Y.; Zheng, B.; Zhu, X.; Wang, X.; Duan, X.; Pan, A. Rational Kinetics Control toward Universal Growth of $2 \mathrm{D}$ Vertically Stacked Heterostructures. Adv. Mater. 2019, 31 (27), 1901351.

(14) Shang, S. L.; Lindwall, G.; Wang, Y.; Redwing, J. M.; Anderson, T.; Liu, Z. K. Lateral Versus Vertical Growth of Two-Dimensional Layered Transition-Metal Dichalcogenides: Thermodynamic Insight into MoS2. Nano Lett. 2016, 16 (9), 5742-50.

(15) Zhang, X.; Nan, H.; Xiao, S.; Wan, X.; Gu, X.; Du, A.; Ni, Z.; Ostrikov, K. K. Transition metal dichalcogenides bilayer single crystals by reverse-flow chemical vapor epitaxy. Nat. Commun. 2019, 10 (1), 598.

(16) Han, A.; Aljarb, A.; Liu, S.; Li, P.; Ma, C.; Xue, F.; Lopatin, S.; Yang, C.-W.; Huang, J.-K.; Wan, Y.; Zhang, X.; Xiong, Q.; Huang, K.W.; Tung, V.; Anthopoulos, T. D.; Li, L.-J. Growth of $2 \mathrm{H}$ stacked WSe2 bilayers on sapphire. Nanoscale Horizons 2019, 4 (6), 14341442.

(17) Jeon, J.; Jang, S. K.; Jeon, S. M.; Yoo, G.; Jang, Y. H.; Park, J. H.; Lee, S. Layer-controlled CVD growth of large-area twodimensional MoS2 films. Nanoscale 2015, 7 (5), 1688-95.

(18) Huang, J.-K.; Pu, J.; Hsu, C.-L.; Chiu, M.-H.; Juang, Z.-Y.; Chang, Y.-H.; Chang, W.-H.; Iwasa, Y.; Takenobu, T.; Li, L.-J. LargeArea Synthesis of Highly Crystalline WSe2Monolayers and Device Applications. ACS Nano 2014, 8 (1), 923-930.

(19) Johari, P.; Shenoy, V. B. Tuning the electronic properties of semiconducting transition metal dichalcogenides by applying mechanical strains. ACS Nano 2012, 6 (6), 5449-5456.

(20) Hsu, W. T.; Lu, L. S.; Wang, D.; Huang, J. K.; Li, M. Y.; Chang, T. R.; Chou, Y. C.; Juang, Z. Y.; Jeng, H. T.; Li, L. J.; Chang, W. H. Evidence of indirect gap in monolayer WSe2. Nat. Commun. 2017, 8 (1), 929.

(21) Desai, S. B.; Seol, G.; Kang, J. S.; Fang, H.; Battaglia, C.; Kapadia, R.; Ager, J. W.; Guo, J.; Javey, A. Strain-induced indirect to direct bandgap transition in multilayer WSe2. Nano Lett. 2014, 14 (8), 4592-7.
(22) Ahn, G. H.; Amani, M.; Rasool, H.; Lien, D.-H.; Mastandrea, J. P.; Ager, J. W., III; Dubey, M.; Chrzan, D. C.; Minor, A. M.; Javey, A. Strain-engineered growth of two-dimensional materials. Nat. Commun. 2017, 8 (1), 608.

(23) Frisenda, R.; Drüppel, M.; Schmidt, R.; Michaelis de Vasconcellos, S.; Perez de Lara, D.; Bratschitsch, R.; Rohlfing, M.; Castellanos-Gomez, A. Biaxial strain tuning of the optical properties of single-layer transition metal dichalcogenides. npj $2 D$ Materials and Applications 2017, 1 (1), 10.

(24) Schmidt, R.; Niehues, I.; Schneider, R.; Drüppel, M.; Deilmann, T.; Rohlfing, M.; De Vasconcellos, S. M.; Castellanos-Gomez, A.; Bratschitsch, R. Reversible uniaxial strain tuning in atomically thin WSe2. 2D Mater. 2016, 3 (2), No. 021011.

(25) Tongay, S.; Suh, J.; Ataca, C.; Fan, W.; Luce, A.; Kang, J. S.; Liu, J.; Ko, C.; Raghunathanan, R.; Zhou, J.; Ogletree, F.; Li, J.; Grossman, J. C.; Wu, J. Defects activated photoluminescence in twodimensional semiconductors: interplay between bound, charged, and free excitons. Sci. Rep. 2013, 3, 2657.

(26) Jones, A. M.; Yu, H.; Ghimire, N. J.; Wu, S.; Aivazian, G.; Ross, J. S.; Zhao, B.; Yan, J.; Mandrus, D. G.; Xiao, D.; Yao, W.; Xu, X. Optical generation of excitonic valley coherence in monolayer WSe2. Nat. Nanotechnol. 2013, 8, 634.

(27) Sharma, R.; Pandey, J.; Sahoo, K. R.; Rana, K. S.; Biroju, R. K.; Theis, W.; Soni, A.; Narayanan, T. N. Spectroscopic correlation of chalcogen defects in atomically thin MoS2 $(1-\mathrm{x})$ Se2x alloys. J. Phys. Mater. 2020, 3 (4), No. 045001.

(28) Lippert, S.; Schneider, L. M.; Renaud, D.; Kang, K. N.; Ajayi, O.; Kuhnert, J.; Halbich, M.-U.; Abdulmunem, O. M.; Lin, X.; Hassoon, K.; Edalati-Boostan, S.; Kim, Y. D.; Heimbrodt, W.; Yang, E.-H.; Hone, J. C.; Rahimi-Iman, A. Influence of the substrate material on the optical properties of tungsten diselenide monolayers. $2 D$ Mater. 2017, 4 (2), No. 025045.

(29) O'Donnell, K.; Chen, X. Temperature dependence of semiconductor band gaps. Appl. Phys. Lett. 1991, 58 (25), 29242926

(30) Tongay, S.; Zhou, J.; Ataca, C.; Lo, K.; Matthews, T. S.; Li, J.; Grossman, J. C.; Wu, J. Thermally driven crossover from indirect toward direct bandgap in 2D semiconductors: MoSe2 versus MoS2. Nano Lett. 2012, 12 (11), 5576-5580.

(31) Niehues, I.; Schmidt, R.; Druppel, M.; Marauhn, P.; Christiansen, D.; Selig, M.; Berghauser, G.; Wigger, D.; Schneider, R.; Braasch, L.; Koch, R.; Castellanos-Gomez, A.; Kuhn, T.; Knorr, A.; Malic, E.; Rohlfing, M.; Michaelis de Vasconcellos, S.; Bratschitsch, R. Strain Control of Exciton-Phonon Coupling in Atomically Thin Semiconductors. Nano Lett. 2018, 18 (3), 1751-1757.

(32) Xu, R.; Pang, F.; Pan, Y.; Lun, Y.; Meng, L.; Zheng, Z.; Xu, K.; Lei, L.; Hussain, S.; Li, Y. J.; Sugawara, Y.; Hong, J.; Ji, W.; Cheng, Z. Atomically Asymmetric Inversion Scales up to Mesoscopic SingleCrystal Monolayer Flakes. ACS Nano 2020, 14 (10), 13834-13840.

(33) Zhang, K.; Hu, S.; Zhang, Y.; Zhang, T.; Zhou, X.; Sun, Y.; Li, T.-X.; Fan, H. J.; Shen, G.; Chen, X.; Dai, N. Self-Induced Uniaxial Strain in MoS2Monolayers with Local van der Waals-Stacked Interlayer Interactions. ACS Nano 2015, 9 (3), 2704-2710.

(34) Aljarb, A.; Cao, Z.; Tang, H. L.; Huang, J. K.; Li, M.; Hu, W.; Cavallo, L.; Li, L. J. Substrate Lattice-Guided Seed Formation Controls the Orientation of 2D Transition-Metal Dichalcogenides. ACS Nano 2017, 11 (9), 9215-9222.

(35) Li, M.-Y.; Shi, Y.; Cheng, C.-C.; Lu, L.-S.; Lin, Y.-C.; Tang, H.L.; Tsai, M.-L.; Chu, C.-W.; Wei, K.-H.; He, J.-H.; Chang, W.-H.; Suenaga, K.; Li, L.-J. Epitaxial growth of a monolayer WSe2-MoS2 lateral $\mathrm{p}-\mathrm{n}$ junction with an atomically sharp interface. Science 2015, 349 (6247), 524-528.

(36) Li, M.-Y.; Pu, J.; Huang, J.-K.; Miyauchi, Y.; Matsuda, K.; Takenobu, T.; Li, L.-J. Self-Aligned and Scalable Growth of Monolayer WSe2-MoS2 Lateral Heterojunctions. Adv. Funct. Mater. 2018, 28 (17), 1706860

(37) Chiu, M.-H.; Tang, H.-L.; Tseng, C.-C.; Han, Y.; Aljarb, A.; Huang, J.-K.; Wan, Y.; Fu, J.-H.; Zhang, X.; Chang, W.-H.; Muller, D. A.; Takenobu, T.; Tung, V.; Li, L.-J. Metal-Guided Selective Growth 
of 2D Materials: Demonstration of a Bottom-Up CMOS Inverter. Adv. Mater. 2019, 31 (18), 1900861.

(38) Hsu, W.-T.; Zhao, Z.-A.; Li, L.-J.; Chen, C.-H.; Chiu, M.-H.; Chang, P.-S.; Chou, Y.-C.; Chang, W.-H. Second harmonic generation from artificially stacked transition metal dichalcogenide twisted bilayers. ACS Nano 2014, 8 (3), 2951-2958.

(39) He, J.; Hummer, K.; Franchini, C. Stacking effects on the electronic and optical properties of bilayer transition metal dichalcogenidesMoS2, MoSe, WS2. Phys. Rev. B: Condens. Matter Mater. Phys. 2014, 89 (7), No. 075409.

(40) Xia, M.; Li, B.; Yin, K.; Capellini, G.; Niu, G.; Gong, Y.; Zhou, W.; Ajayan, P. M.; Xie, Y.-H. Spectroscopic Signatures of $\mathrm{AA}^{\prime}$ and AB Stacking of Chemical Vapor Deposited Bilayer MoS2. ACS Nano 2015, 9 (12), 12246-12254.

(41) Pimpinelli, A.; Villain, J. Physics of Crystal Growth; Cambridge University Press, 1999. DOI: 10.1017/CBO9780511622526

(42) Oura, K.; Lifshits, V.; Saranin, A.; Zotov, A.; Katayama, M. Surface Science: An Introduction; Springer Science \& Business Media, 2013.

(43) Aljarb, A.; Fu, J.-H.; Hsu, C.-C.; Chuu, C.-P.; Wan, Y.; Hakami, M.; Naphade, D. R.; Yengel, E.; Lee, C.-J.; Brems, S.; Chen, T.-A.; Li, M.-Y.; Bae, S.-H.; Hsu, W.-T.; Cao, Z.; Albaridy, R.; Lopatin, S.; Chang, W.-H.; Anthopoulos, T. D.; Kim, J.; Li, L.-J.; Tung, V. Ledgedirected epitaxy of continuously self-aligned single-crystalline nanoribbons of transition metal dichalcogenides. Nat. Mater. 2020, 19 (12), 1300-1306.

(44) Unuchek, D.; Ciarrocchi, A.; Avsar, A.; Watanabe, K.; Taniguchi, T.; Kis, A. Room-temperature electrical control of exciton flux in a van der Waals heterostructure. Nature 2018, 560 (7718), 340-344.

(45) Chiu, M. H.; Zhang, C.; Shiu, H. W.; Chuu, C. P.; Chen, C. H.; Chang, C. Y.; Chen, C. H.; Chou, M. Y.; Shih, C. K.; Li, L. J. Determination of band alignment in the single-layer MoS2/WSe2 heterojunction. Nat. Commun. 2015, 6, 7666.

(46) Kresse, G.; Furthmüller, J. Efficiency of ab-initio total energy calculations for metals and semiconductors using a plane-wave basis set. Comput. Mater. Sci. 1996, 6 (1), 15-50.

(47) Perdew, J. P.; Burke, K.; Ernzerhof, M. Generalized gradient approximation made simple. Phys. Rev. Lett. 1996, 77 (18), 3865.

(48) Klimeš, J.; Bowler, D. R.; Michaelides, A. Van der Waals density functionals applied to solids. Phys. Rev. B: Condens. Matter Mater. Phys. 2011, 83 (19), 195131. 